\title{
The local transcriptional regulators SacR1 and SacR2 act as repressors of fructooligosaccharides metabolism in Lactobacillus plantarum
}

\author{
Chen Chen, Linlin Wang, Haiyan Yu and Huaixiang Tian * (1)
}

\begin{abstract}
Background: In Lactobacillus plantarum, fructooligosaccharides (FOS) metabolism is controlled by both global and local regulatory mechanisms. Although catabolite control protein A has been identified as a global regulator of FOS metabolism, the functions of local regulators remain unclear. This study aimed to elucidate the roles of two local regulators, SacR1 and SacR2, in the regulation of FOS metabolism in L. plantarum both in vitro and in vivo.

Results: The inactivation of sacR1 and sacR2 affected the growth and production of metabolites for strains grown on FOS or glucose, respectively. A reverse transcription-quantitative PCR analysis of one wild-type and two mutant strains ( $\triangle s a c R 1$ and $\triangle s a c R 2$ ) of L. plantarum identified SacR1 and SacR2 as repressors of genes relevant to FOS metabolism in the absence of FOS, and these genes could be induced or derepressed by the addition of FOS. The analysis predicted four potential transcription factor binding sites (TFBSS) in the putative promoter regions of two FOS-related clusters. The binding of SacR1 and SacR2 to these TFBSs both in vitro and in vivo was verified using electrophoretic mobility shift assays and chromatin immunoprecipitation, respectively. A consensus sequence of WNNNNNAACGNNTTNNNNNW was deduced for the TFBSs of SacR1 and SacR2.
\end{abstract}

Conclusion: Our results identified SacR1 and SacR2 as local repressors for FOS metabolism in L. plantarum. The regulation is achieved by the binding of SacR1 and SacR2 to TFBSs in the promoter regions of FOS-related clusters. The results provide new insights into the complex network regulating oligosaccharide metabolism by lactic acid bacteria.

Keywords: Lactobacillus plantarum, Fructooligosaccharides, Local regulatory mechanism, Transcriptional factors binding sites, Regulatory network

\section{Background}

Lactobacillus plantarum is a Gram-positive bacterium that resides naturally in the human gastrointestinal tract (GIT) [1, 2]. This species is a common and versatile type of lactic acid bacteria (LAB) used in the production of several fermented and functional foods $[1,3-5]$. Like most lactobacilli, L. plantarum strains have complex

*Correspondence: tianhx@sit.edu.cn

School of Perfume and Aroma Technology, Shanghai Institute

of Technology, Shanghai 201418, People's Republic of China nutritional requirements for fermentable carbohydrates and can utilize a wide range of carbohydrates, including some prebiotics $[4,6,7]$. Fructooligosaccharides (FOS) are non-digestible food ingredients that can selectively stimulate the growth and activity of beneficial intestinal microbiota and are considered an established type of prebiotic $[4,8,9]$. Several studies have demonstrated that L. plantarum can effectively utilize FOS [10-12]. This advantage helps the survival and colonization of L. plantarum in the GIT $[1,6,8]$. 
In bacteria, the uptake and assimilation of different carbohydrates are tightly regulated, as the simultaneous utilization of all accessible sugars would be energetically inefficient [13]. The presence of preferred carbon sources prevents the utilization of secondary substrates via a phenomenon called carbon catabolite repression (CCR) $[14,15]$. CCR, a complex regulatory phenomenon, is frequently mediated by several mechanisms [16] that either affect the synthesis of catabolic enzymes via global or specific regulators or inhibit the uptake of a carbon source and, consequently, the formation of the corresponding inducer [17]. According to previous reports, carbohydrate utilization by lactobacilli is always subject to CCR, which is achieved via the combined effects of global and operon-specific (i.e., local) regulatory mechanisms $[18,19]$. Regarding the former type of regulatory mechanism, catabolite control protein A (CcpA) affects global transcriptional control by binding to catabolite repression element ( $\mathrm{cre}$ ) sites located in or downstream of the putative -35 and -10 sequences in the presence of more favorable carbon sources [20-24]. Regarding the latter, local regulons generally control a small number of genes and operons that are combined with specific operator motifs in the absence of the related substrate $[25,26]$. Specifically, studies of the metabolic regulation of oligosaccharide utilization, such as FOS [27] and galactooligosaccharides (GOS) [28], have identified additional potential global and local regulatory factors in LAB, indicating that gene clusters associated with metabolic oligosaccharides are under the dual role of global and local regulation.

Previously, we identified two gene clusters, sacPTS1 and sacPTS26, that are involved in the utilization of FOS in L. plantarum [11, 12]. The sacPTS1 cluster is composed of five genes that encoded a sucrose phosphoenolpyruvate transport system (PTS1), a fructofuranosidase (SacA), a fructokinase (SacK), an $\alpha$-glucosidase (Agl2), and a repressor (SacR1). The sacPTS26 cluster encodes a sucrose PTS (PTS26), an $\alpha$-glucosidase (Agl4), and a transcriptional regulator (SacR2) [11]. Specifically, two genes encoding the assumed repressor protein, sacR1

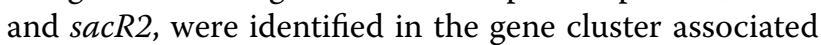
with FOS metabolism and found to exhibit significant similarity to members of the GalR-LacI family of bacterial transcription regulators [11]. Subsequently, we demonstrated that CcpA is a vital regulator of FOS metabolism in L. plantarum, and it functions through the direct binding toward the cre sites in the promoter regions of FOSrelated clusters [12]. However, the mechanism by which FOS metabolism is regulated via local regulators in $L$. plantarum remains unclear.

To determine whether FOS metabolism in L. plantarum is regulated locally by CCR, we firstly compared

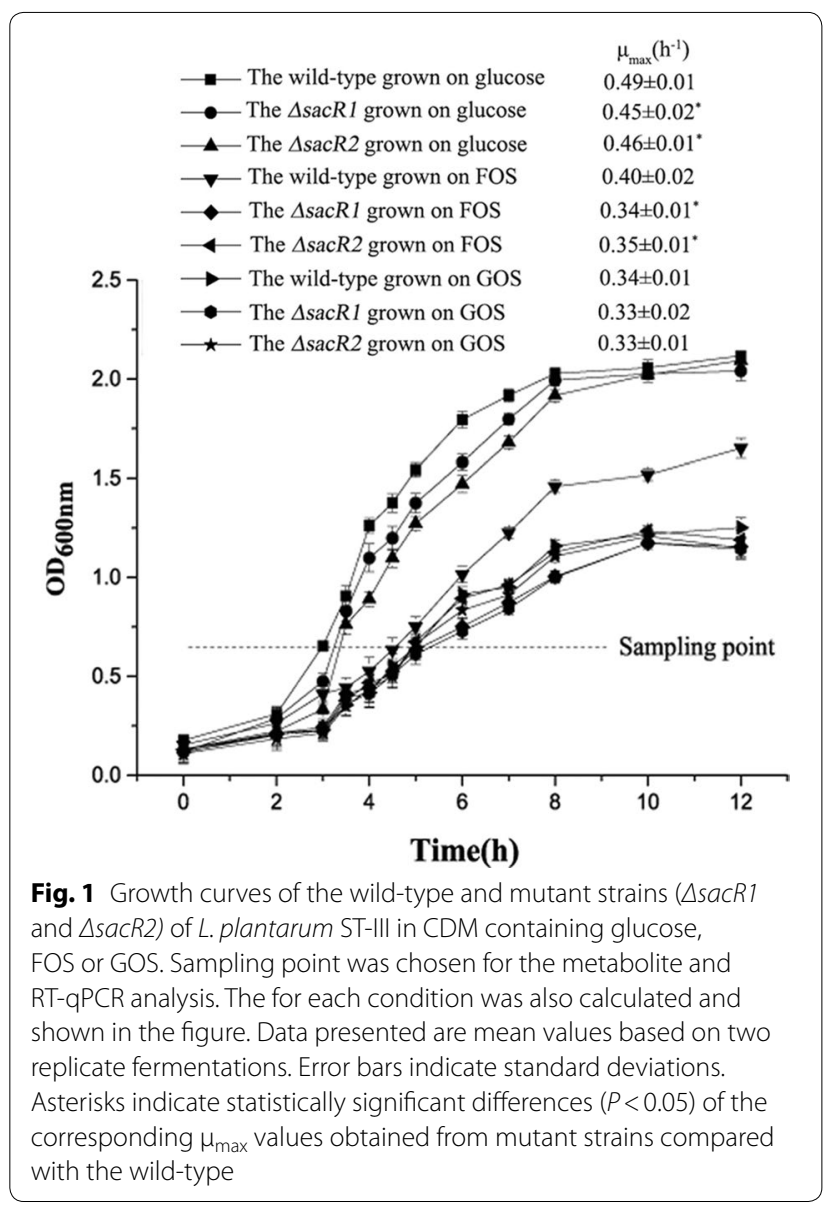

the physiological states of $L$. plantarum and mutant strains via growth profiles and metabolite production analysis. We then used reverse transcription-quantitative PCR (RT-qPCR) to compare the expression of relevant genes in cultures grown in chemically defined medium (CDM) with different sugars $[29,30]$. Moreover, we predicted the presumed binding sites of local regulators in $L$. plantarum and verified these sites using electrophoretic mobility shift assays (EMSAs) and chromatin immunoprecipitation (ChIP) to detect in vitro and in vivo interactions, respectively. The results of this study shed new light on the network that regulates FOS metabolism in L. plantarum and reveals the essential roles of operonspecific transcriptional regulators in the control of FOS utilization.

\section{Results \\ Growth profiles and metabolite production of the wild-type and mutant strains}

To determine the functions of SacR1 and SacR2 in FOS utilization, two mutant strains $(\triangle s a c R 1$ and $\triangle s a c R 2)$ were constructed using the Cre-lox-based mutagenesis 
Table 1 Comparison of metabolites resulting from the fermentation of glucose, FOS and GOS by wild-type L. plantarum $\mathrm{ST}$-III and $\Delta s a c R 1$ and $\Delta s a c R 2$ strains at $\mathrm{OD}_{600}$ of 0.65

\begin{tabular}{|c|c|c|c|c|c|c|c|c|c|}
\hline & \multicolumn{3}{|c|}{ Wild-type strain } & \multicolumn{3}{|l|}{$\Delta s a c R 1$ strain } & \multicolumn{3}{|l|}{$\Delta s a c R 2$ strain } \\
\hline & Lactate & Acetate & Formate & Lactate & Acetate & Formate & Lactate & Acetate & Formate \\
\hline Glucose & $15.60 \pm 0.19$ & $3.17 \pm 0.06$ & $1.64 \pm 0.10$ & $15.01 \pm 0.07^{\#}$ & $2.95 \pm 0.02^{\#}$ & $1.48 \pm 0.05^{\#}$ & $15.04 \pm 0.04^{\#}$ & $2.88 \pm 0.02^{\#}$ & $1.43 \pm 0.01^{\#}$ \\
\hline $\mathrm{FOS}^{\mathrm{a}, \mathrm{b}}$ & $12.34 \pm 0.17^{*}$ & $5.63 \pm 0.10^{*}$ & $1.47 \pm 0.15^{*}$ & $11.09 \pm 0.05^{*, \#}$ & $4.35 \pm 0.03^{*, \#}$ & $1.23 \pm 0.14^{*, \#}$ & $10.90 \pm 0.38^{*, \#}$ & $4.21 \pm 0.12^{*, \#}$ & $1.26 \pm 0.10^{*, \#}$ \\
\hline GOS & $12.28 \pm 0.10^{*}$ & $6.08 \pm 0.15^{*}$ & $1.49 \pm 0.06^{*}$ & $12.19 \pm 0.01^{*}$ & $6.26 \pm 0.11^{*}$ & $1.46 \pm 0.06$ & $12.18 \pm 0.39^{*}$ & $6.11 \pm 0.15^{*}$ & $1.43 \pm 0.21^{*}$ \\
\hline
\end{tabular}

Data presented are mean values based on two replicate fermentations. Error bars indicate standard deviations

a Asterisks indicate statistically significant differences $(P<0.05)$ of the corresponding values obtained from cells grown on FOS or GOS compared with those grown on glucose

b Octothorpes indicate statistically significant differences $(P<0.05)$ of the corresponding values obtained from $\Delta s a c R 1$ or $\Delta s a c R 2$ mutant compared with those of wildtype

system [31]. As the presence of glucose might induce global regulation [32, 33], which would mask the effect of local regulation [34]. We also selected another oligosaccharide-GOS that is not related to the FOS metabolism pathway for analysis and comparison [28, 35]. The growth of these mutant strains on glucose, FOS and GOS was compared with that of the wild-type strain (Fig. 1). The values of maximal growth rate $\left(\mu_{\max }\right)$ were significantly higher for the wild-type than the mutant strain $(P<0.05)$ in the logarithmic phase for glucose and FOS. For the strains grown on GOS, the growth curves were almost the same, and no difference was observed among their $\mu_{\max }$ values $(P>0.05)$.

Our past findings clearly indicated that the metabolites of L. plantarum are mainly lactate, acetate and formate $[10-12,35]$. Therefore, we also determined the levels of organic acids generated during fermentation with $L$. plantarum ST-III and two mutant strains on glucose, FOS or GOS, respectively (Table 1). Lactate and acetate are the main end products, resulting from the fermentation of the three carbon sources. Wildtype and the two mutant strains grown on FOS or GOS produced less lactate and more acetate than grown on glucose $(P<0.05)$. The results suggest that a shift from homolactic fermentation to mixed fermentation has occurred, which is consistent with our previous results $[12,35]$. In the absence of SacR1 and SacR2, the metabolic products decreased compared with corresponding values for the wild-type strain grown on FOS $(P<0.05)$. This situation also occurred in the presence of glucose $(P<0.05)$, although to a lesser extent. The levels of metabolites did not vary between the wildtype and two mutant strains grown on GOS. These results are also in agreement with the growth profiles for the wild-type and two mutant strains. These data suggest that the inactivation of sacR1 and sacR2 impairs the growth and the metabolite formation of L. plantarum in cultures containing FOS or glucose, respectively.

\section{RT-qPCR revealed repressor roles of SacR1 and SacR2}

The differential expression of relevant genes was studied in comparison with growth on FOS and GOS through an RT-qPCR analysis. Our previous results showed that FOS is transported intact across the membrane by two PTSs (PTS1 and PTS26) and hydrolyzed by SacA into fructose and glucose-6-phosphate. Fructose is converted to fructose 6-phosphate under the action of SacK [11, 12]. Thus, the $\mathrm{C}_{\mathrm{T}}$ values for these three genes, $s a c K, s a c A$, and pts26, were selected as the key genes for FOS metabolism

Table 2 Relative transcript abundances of FOS-related genes in the wild-type and $\Delta$ sacR1 and $\Delta$ sacR2 strains grown in different sugars

\begin{tabular}{|c|c|c|c|c|c|c|}
\hline \multirow[t]{2}{*}{ Gene } & \multicolumn{2}{|c|}{ Wild-type strain } & \multicolumn{2}{|c|}{$\Delta s a c R 1$ strain } & \multicolumn{2}{|c|}{$\Delta s a c R 2$ strain } \\
\hline & FOS & GOS & FOS & GOS & FOS & GOS \\
\hline sacPTS26 $6^{a, b}$ & $3.10 \pm 0.32^{*}$ & $1.51 \pm 0.17$ & $3.14 \pm 0.18^{*}$ & $1.22 \pm 0.05$ & $3.04 \pm 0.31$ & $3.27 \pm 0.16$ \\
\hline$s a c A^{a, b}$ & $3.35 \pm 0.29^{*}$ & $1.28 \pm 0.23$ & $3.18 \pm 0.14$ & $3.24 \pm 0.24$ & $3.42 \pm 0.12^{*}$ & $1.11 \pm 0.09$ \\
\hline$s a c k^{a, b}$ & $3.16 \pm 0.36^{*}$ & $1.05 \pm 0.19$ & $3.02 \pm 0.22$ & $3.08 \pm 0.27$ & $2.95 \pm 0.31^{*}$ & $0.87 \pm 0.14$ \\
\hline
\end{tabular}

Data presented are mean values based on at least three replicates. Error bars indicate standard deviations

${ }^{a}$ The relative transcription abundances of each gene in different conditions were calculated by the $2^{-\triangle \mathrm{Ct}}$ method and $16 \mathrm{~S}$ rRNA was used as the internal standard

b Asterisks indicate statistically significant differences $(P<0.05)$ of the corresponding values obtained from cells grown on FOS compared with those grown on GOS 


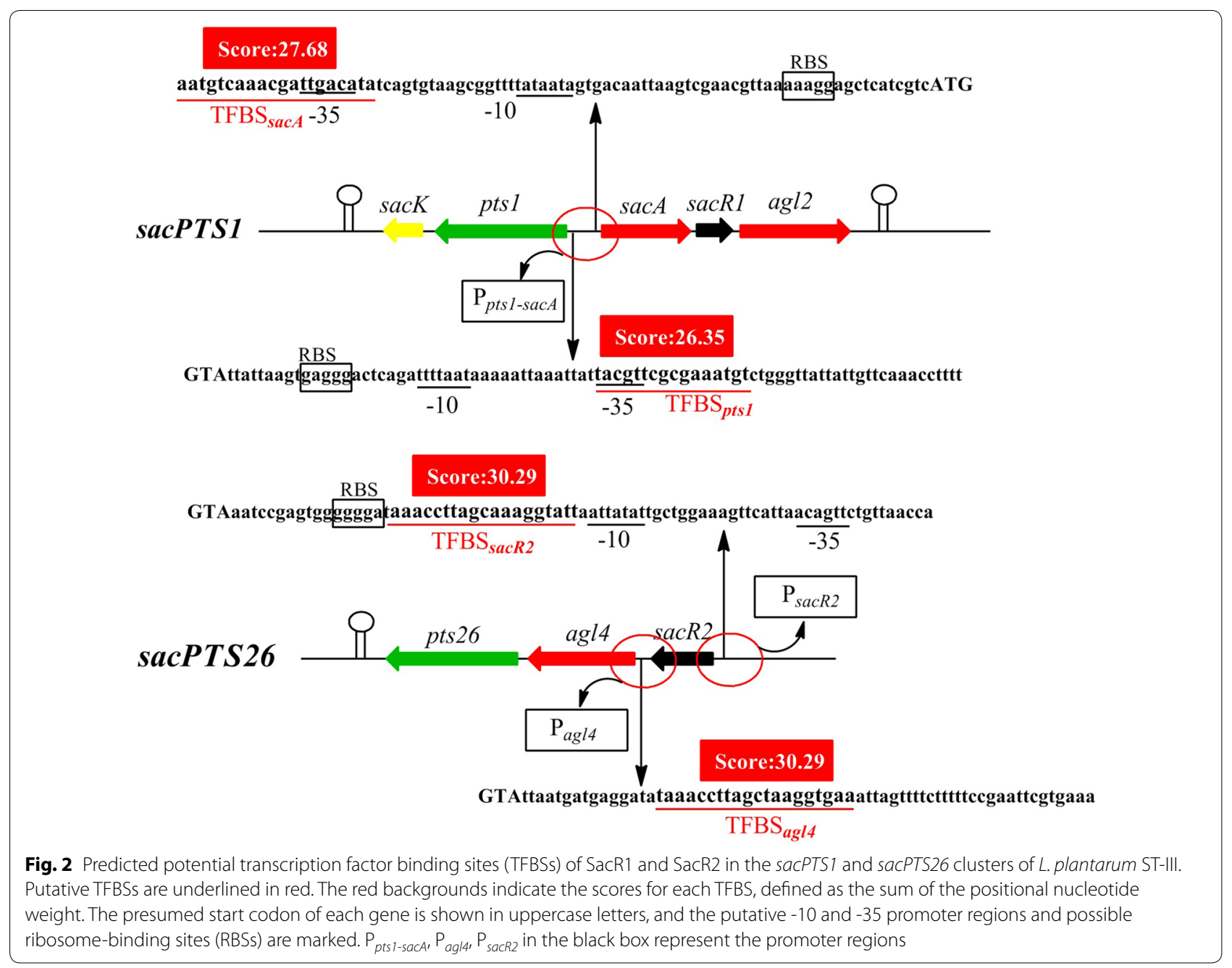

and used to calculate the fold changes between conditions. As expected, the expression of all three genes was significantly up-regulated in the wild-type strain in the presence of FOS relative to GOS $(P<0.05$, Table 2$)$. In contrast, after $s a c R 1$ and $s a c R 2$ inactivation, the induction or derepression of the expression of these genes in response to FOS was nearly absent $(P>0.05$, Table 2$)$. For example, the levels of $s a c A$ and $s a c K$ expression in the wild-type strain exposed FOS were 2.62 and 3.01-fold higher, respectively, than those in wild-type cells exposed to GOS. In contract, the expression levels of these two genes were roughly the same in the presence of FOS and GOS to the $\triangle s a c R 1$ strain. Similar expression patterns were also observed for the sacPTS26 operons and in the $\triangle s a c R 2$ strain. These results verify that SacR1 and SacR2 repress expression of genes relevant to FOS metabolism in the absence of FOS, FOS metabolism could be induced or derepressed by the addition of FOS.

\section{Analysis of binding site consensus}

Local regulators can regulate target genes by interacting with specific transcription factor binding site (TFBS) in the operon [36, 37]. However, the binding sites used by SacR1 and SacR2 had not previously been elucidated. Accordingly, we searched the RegPrecise database for a conserved common binding consensus motif based on the profiles of TFBSs of local regulators in L. plantarum WCSF1. First, a positional frequency matrix (PFM) was constructed according to the frequency of occurrence of each base at each location of the consensus sequence (Fig. 2). Next, the generated PFM was used to search the sacPTS1 and sacPTS26 clusters, where two potential TFBSs were identified in the $\mathrm{P}_{\text {pts1-sacA }}$ region (TFBS-1, AATGTCAAACGATTGACATA; TFBS-2, TACGTT CGCGAAATGT). Additionally, one binding site each was identified in the $\mathrm{P}_{\text {sack-agl }}$ region (TFBS-3, TAAACC TTAGCTAAGGTGAA) and the $\mathrm{P}_{\text {sack }}$ region (TFBS-4, 


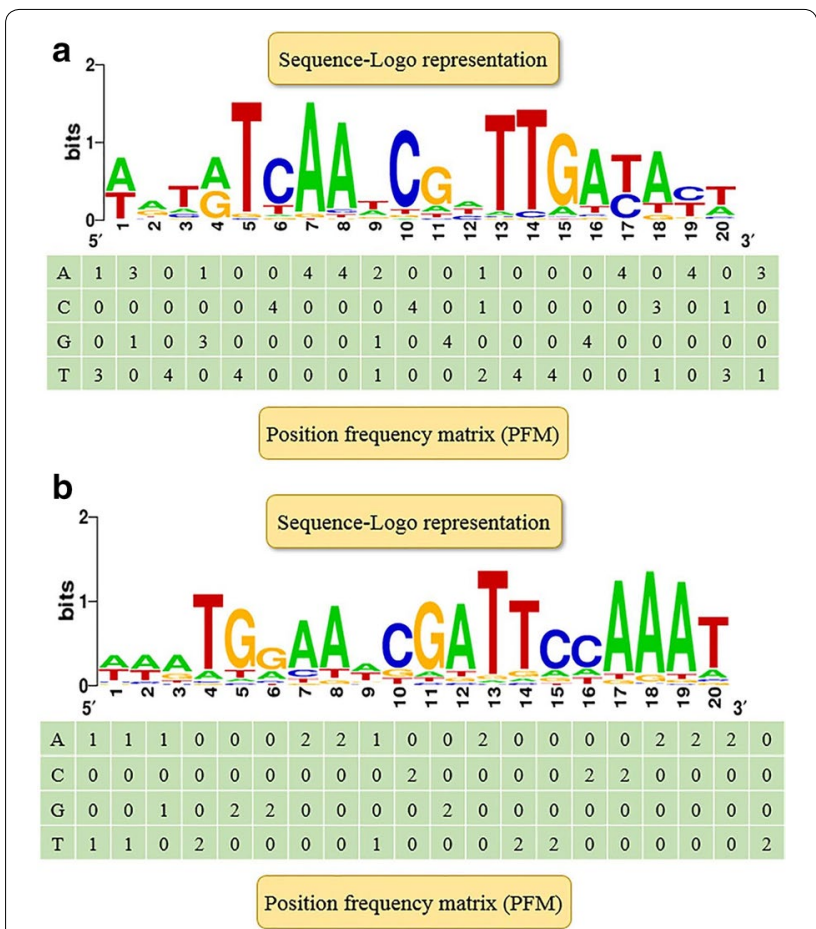

Fig. 3 Consensus sequence motif of the transcription factor binding sites (TFBSs) in L. plantarum ST-III, generated using RSAT software. A positional frequency matrix (PFM) was generated according to the frequency of occurrence of each base at each location of the consensus sequence. The sequence-logo represents the occurrence frequency, and the height of each individual symbol reflects its prevalence at a given position. a Consensus sequence motif of the $\mathrm{SacR} 1 ; \mathbf{b}$ Consensus sequence motif of the SacR2

AAACCTTAGCAAAGGTATT) (Fig. 3). The scores of these four candidate sites were all $>5$, suggesting SacR1 and SacR2 binding [38].

\section{Confirmation of the binding of local regulators to sequence motifs in vitro}

We next performed EMSAs to identify the four putative TFBSs to which the SacR1 and SacR2 proteins bind specifically in vitro $[39,40]$. For the first step, both proteins were expressed successfully in different recombinant strains (BL21-sacR1 and BL21-sacR2) and purified. Then the purified protein was used to perform EMSAs with the DNA probes generated in the possible promoter regions of the sacPTS1 and sacPTS26 clusters. As shown in Fig. 4a, c, e (lanes 1-4), the amounts of the SacR1-DNA and SacR2-DNA complexes increased with increasing concentrations of $\mathrm{His}_{6}$-tagged SacR1 $(0-3 \mu \mathrm{g})$ and $\mathrm{His}_{6}$-tagged SacR2 $(0-10 \mu \mathrm{g})$ proteins. In contrast, when labeled and unlabeled probes were used in a specific competitive assay (lane 5), no shift was detected for the labeled probe, indicating the binding specificities of SacR1 and SacR2 for these DNA fragments. Furthermore, except for the binding shown in Fig. 4, SacR1 cannot bind to the putative TFBSs of SacR2, and vice versa (data not shown).

Next, to verify the specific binding of SacR1 and SacR2 to the TFBSs, each putative TFBS which was generated from Regulatory Sequence Analysis Tools (RSAT) analysis according to the consensus motif, was mutated and named as TFBS-MUT. The main principle of the mutation was as follows: the defined base in the consensus motif was mutated to the other three bases, and the "W" that represents A or T was mutated to "S", which represents $G$ or $C[33,40]$. DNA fragments of the three promoter regions containing the TFBS-MUT sites were generated by PCR and used in EMSAs [41]. Notably, the binding of $\mathrm{His}_{6}$-tagged SacR2 to the mutant $\mathrm{P}_{a g l 4}$ and $\mathrm{P}_{\text {sacR } 2}$ regions was completely abolished (Fig. $4 \mathrm{~d}, \mathrm{f}$ ). As the $\mathrm{P}_{p t s 1-s a c A}$ region exists at two putative binding sites, it was mutated twice prior to the EMSA. The binding affinity of $\mathrm{His}_{6}$-tagged SacR1 protein for the $\mathrm{P}_{p t s 1-s a c A}$ region was weakened after a single mutation $\left(\mathrm{TFBS}_{s a c A}\right.$; data not shown). After the double mutation, $\mathrm{His}_{6}$-tagged SacR1 could no longer bind to the new $\mathrm{P}_{\text {pts1-sacA }}$ region (Fig. 4b). In conclusion, these results indicate that SacR1 and SacR2 proteins could bind specifically to the putative TFBSs in the sacPTS1 and sacPTS26 clusters, respectively.

\section{Confirmation of the binding of local regulators to the sequence motifs in vivo}

Next, we performed a ChIP-qPCR analysis to validate the predicted interactions of SacR1 and SacR2 proteins with TFBSs in vivo. SacR1 and SacR2 were labeled with $\mathrm{N}$-terminal FLAG-tags, and the subsequent successful expression of 409-Flag-sacR1 and 409-Flag-sacR2 in L. plantarum was confirmed via a western blot analysis (Fig. 5a). Next, both the ChIP-extracted and input DNA were examined by qPCR. As shown in Fig. 5b, the fragments TFBS pts $1_{1}$ and TFBS sacA $_{\text {a }}$ were remarkably enriched (22.0 and 28.1-fold, respectively) by IP with the FLAGtagged SacR1 protein when compared with mock ChIP samples, demonstrating that SacR1 interacts specifically with the $\mathrm{P}_{p t s 1-s a c A}$ region in vivo. Similarly, the fragments $\mathrm{TFBS}_{a g l 4}$ and $\mathrm{TFBS}_{\text {sacR2 }}$ were also remarkably enriched (6.2- and 20.9-fold, respectively) by IP with FLAG-tagged SacR2. No other enrichment was observed in Fig. 5, which once again proved that SacR1 cannot bind to the TFBSs of SacR2, as is the case for SacR2. Together, these findings suggest that SacR1 and SacR2 can bind 

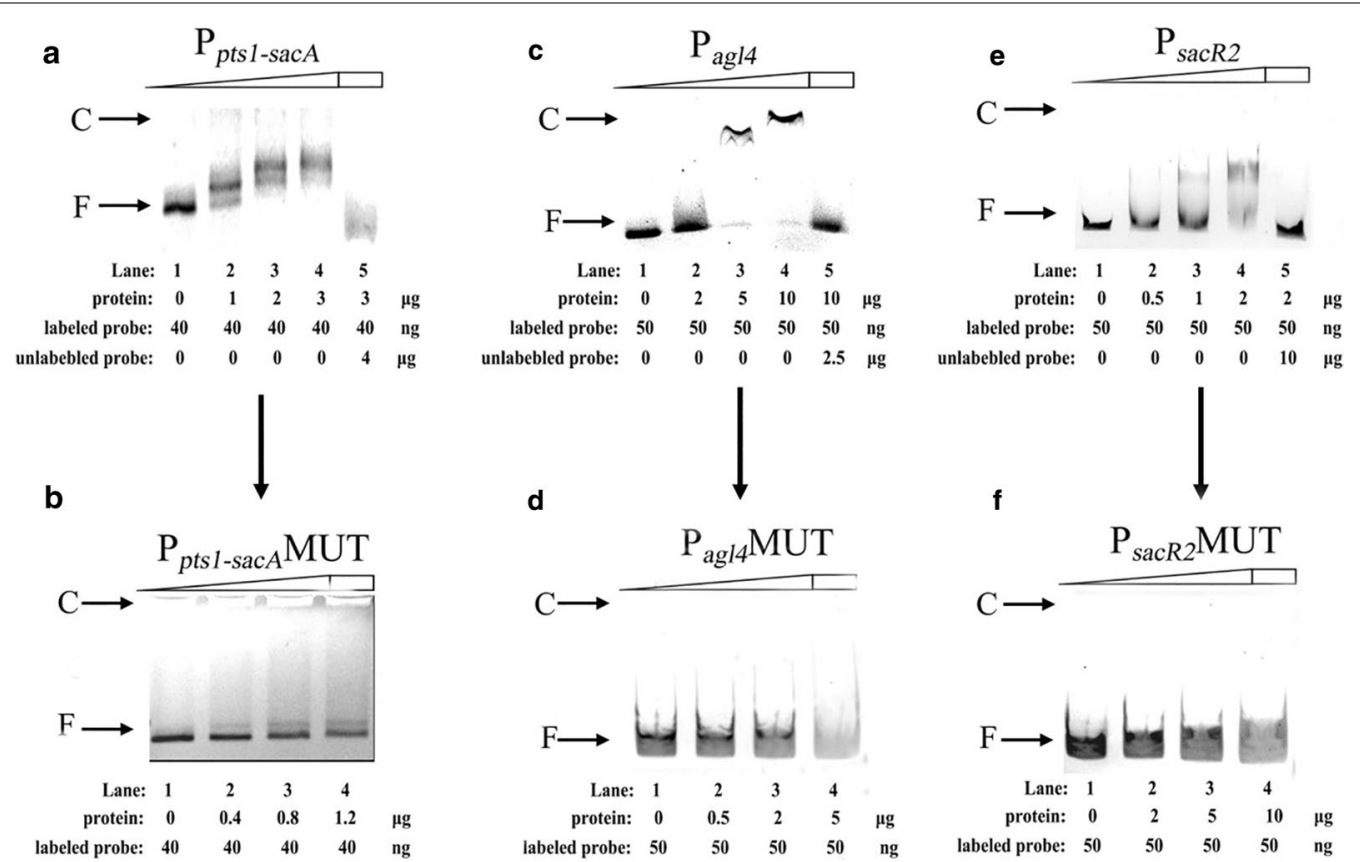
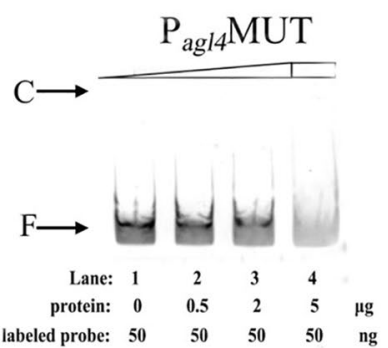
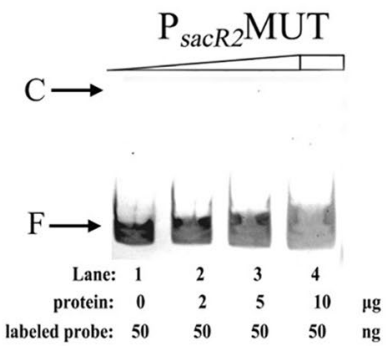

Fig. 4 Characterization and verification of SacR1-DNA and SacR2-DNA binding at the four promoter regions by electrophoretic mobility shift

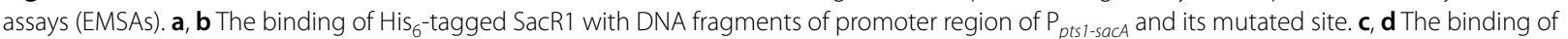
His $_{6}$-tagged SacR2 with DNA fragments of promoter region of $\mathrm{P}_{\text {agl }}$ and its mutated site. $\mathbf{e}$, $\mathbf{f}$ The binding of His ${ }_{6}$-tagged SacR2 with DNA fragments of promoter region of $P_{S a c R 2}$ and its mutated site. The positions of the SacR1-DNA and SacR2-DNA complexes (c) or free DNA (f) are indicated at the left of the figure

specifically to their corresponding TFBSs in the identified promoter regions of the two clusters.

\section{Discussion}

L. plantarum is a versatile species that can grow on numerous types of carbohydrates. Notably, this bacterial species can utilize FOS, although in a relatively low efficiency compared with glucose, and harbors two gene clusters that participate in FOS metabolism [11]. Due to the complex interspecies competition in the GIT, the regulation of FOS metabolism is crucial to the survival and colonization of L. plantarum. We have verified that CcpA, a GalR-LacI family protein, is a vital regulator of FOS metabolism in L. plantarum; two local regulators, SacR1 and SacR2, are involved in the regulation of these two FOS metabolism-related clusters [12]. These results suggested that the utilization of FOS in L. plantarum may involve the double effects of global and local regulation. However, the specific manner by which SacR1 and SacR2 control local regulation have not been determined. In this report, we evaluated the regulation of FOS metabolism by local regulatory elements in L. plantarum both in vitro and in vivo.

The CCR in response to glucose may have been predominant in the context of dual regulation, whereas the effects of local regulators could not be observed [18]. Thus, we also included GOS as an alternative carbon source to verify the roles of SacR1 and SacR2. Combining the results of growth profiles, metabolite production and gene expression, we found that inactivation of these two local regulators significantly affected growth and fermentation end-products in L. plantarum and they acted as repressors of FOS-related genes in the absence of FOS. The deletion of sacR 1 and sacR 2 could also affect the growth and metabolite formation for strains grown on glucose, but to a lesser extent. These two regulators did not affect the metabolism of GOS. These results revealed the regulatory complexity for sugar utilization in $L$. plantarum.

The regulation of locally regulated gene transcription involves the binding of specific regulators to binding sites on the target genes [25, 26]. However, potential SacR1 and SacR2 binding sequences had not previously been clarified in the two FOS metabolism-related clusters in L. plantarum. In this study, we identified four putative TFBSs in the promoter regions of FOS-related clusters. These sites were predicted based on the consensus motif generated from RSAT analysis. Then the specific binding interactions in vitro and in vivo were verified in this study by EMSA and ChIP-qPCR, respectively. Although both regulators showed a low level of sequence identity (28\%), 

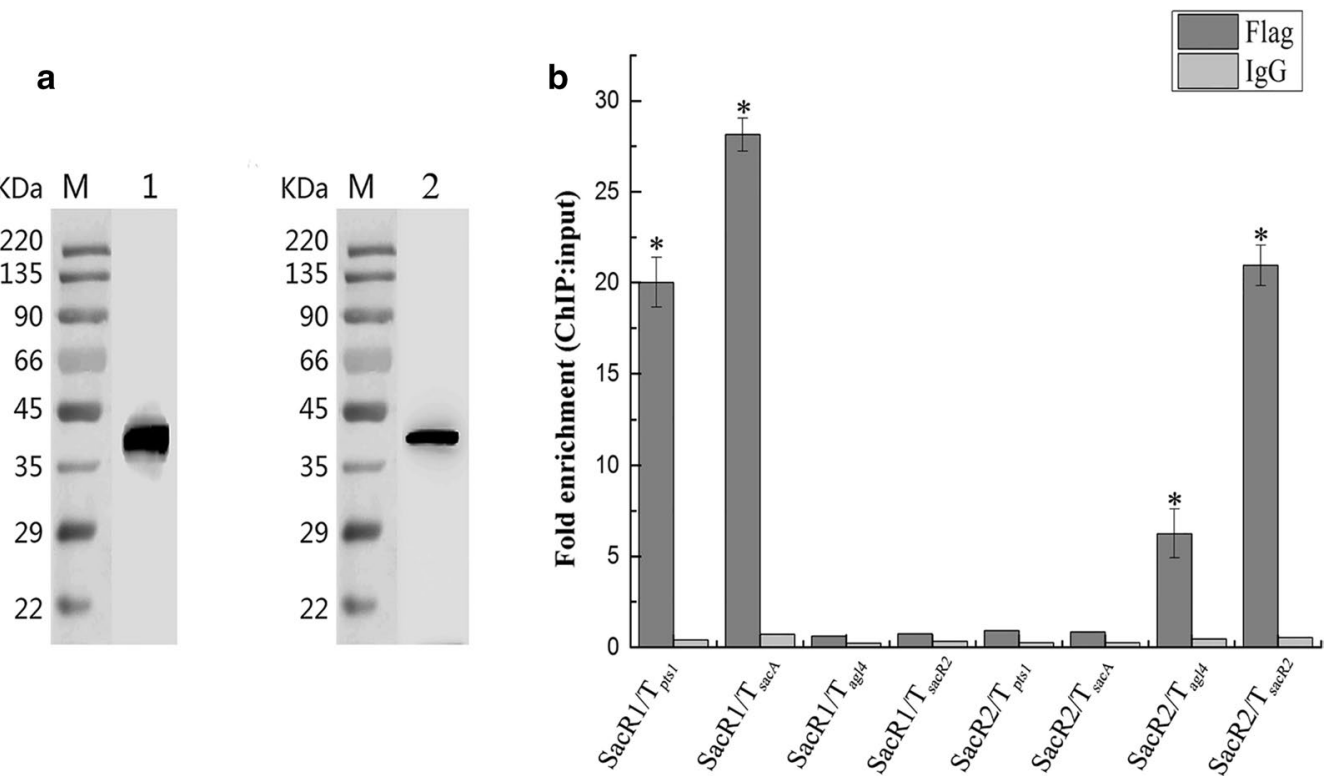

Fig. 5 Chromatin immunoprecipitation (ChIP) analysis of the binding of SacR1 and SacR2 to the transcription factor binding sites (TFBSs) in the three promoter regions. a Detection of FLAG-tagged SacR1 and SacR2 proteins by western blotting with a FLAG-specific antibody. 1, SacR1; 2,

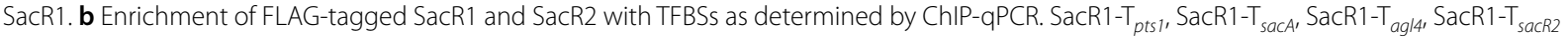

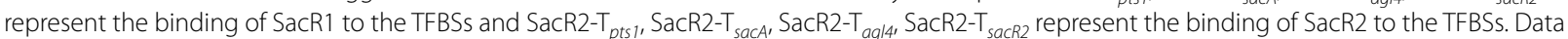
are presented as mean values based on at least three replicates. Error bars indicate standard deviations. Values that differed significantly $(P<0.05)$ from those of the negative control (normal rabbit lgG) are indicated with asterisks

they both belong to the GalR-LacI family of CcpA-like proteins and are expected to have similar DNA-binding features $[11,26]$. When combining the predicted TFBSs of SacR1 and SacR2 with our present results, we deduced a consensus sequence for the SacR1 and SacR2 binding sites, WNNNNNAACGNNTTNNNNNW ( $=$ any base, $\mathrm{W}=\mathrm{A}$ or $\mathrm{T}$ ), which is also similar to the consensus sequence of cre sites [19, 26]. However, no cross reaction was observed for SacR1 and SacR2 with other TFBSs. A possible reason is that although TFBSs of SacR1 and SacR2 are similar in structure, some differences in the sequences may hinder the binding by other regulators. These results provide a new insight into the structures of local regulator recognition sites in Gram-positive bacteria. Related foot-printing and CHIP-seq experiments to confirm the binding of SacR1 and SacR2 to the target sites are ongoing.

Many studies revealed a double effect of global and local regulation on carbohydrate metabolism in LAB [42, 43]. In contrast to these global regulators, local regulators regulate only one or a few genes that are often linked genetically to the gene encoding the regulator itself [44]. For instance, Tamara et al. [45] identified a novel RpiRfamily transcription activator, GlaR, positioned directly upstream of the gal-lac gene cluster in Lactococcus lactis IL1403. GlaR was identified as a transcriptional activator of galactose and lactose utilization genes, the expression of which can be induced by galactose. Moreover, six LacIfamily local transcriptional factors and a TetR-family regulator were identified as presumptive local repressors of arabino-oligosaccharide (AOS) utilization in Bifidobacterium species [46]. According to our previous studies and the present work, FOS metabolism is regulated both globally and locally in L. plantarum [11, 12]. Regulation can be divided into four conditions based on the available carbon source, as follows: only glucose, only FOS, both glucose and FOS, and neither glucose nor FOS. These conditions enable the deduction of the possible regulatory mode. If only glucose is present (Fig. 6a), the binding of CcpA to cre sites would block the transcription of FOS-related genes in L. plantarum. If only FOS is present (Fig. 6c), FOS would bind to repressor proteins (SacR1 and SacR2) to reverse the inhibition induced by the binding of SacR1 and SacR2 to TFBSs in the promoter regions of FOS-related clusters. If neither source is present (Fig. 6d), SacR1 and SacR2 act as repressors and inhibit the expression of FOS-related clusters. If both sources are present (Fig. 6b), FOS acts as an inducer, thus rendering the repressor proteins allosteric and releasing inhibition; however, the global regulator CcpA binds to cre sites and thus remains capable of eliciting CcpA-mediated CCR. This latter process is also the cause of the diauxic growth 


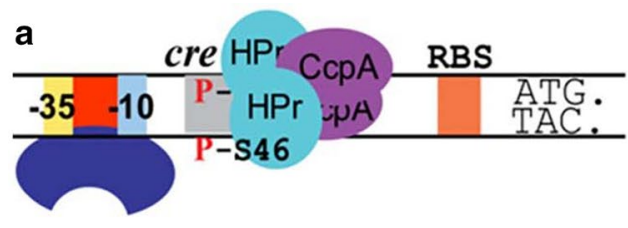

glucose+FOS-
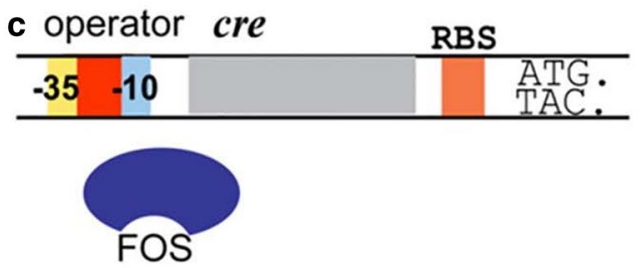

glucose-FOS + b
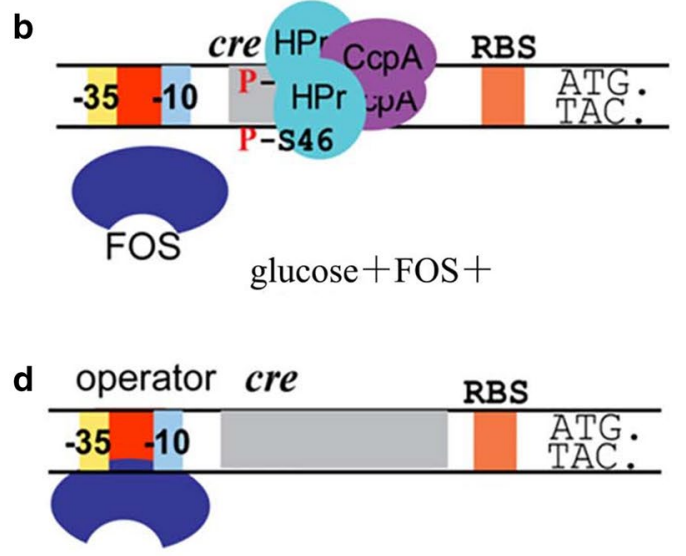

glucose - FOS -

Fig. 6 Mechanisms underlying the global and local regulation of FOS metabolism in L. plantarum. a Presence of glucose. b Presence of both glucose and FOS. c Presence of FOS. $\mathbf{d}$ Absence of glucose and FOS

phenomenon, in which cells resume growing and enter a second growth phase fueled by FOS as the carbon source once glucose is depleted [12]. However, the actual mechanism of regulation may be more complex, as these local regulators are also activated or repressed by CcpA [10, 12]. Furthermore, SacR1 and SacR2 are co-transcribed with other FOS-related genes, suggesting that both proteins act as self-regulators to maintain their own expression [10]. In summary, FOS metabolism is an extremely complex network in which the combined actions of global and local regulators orchestrate the transcription of various units that enable bacteria to adjust sugar utilization to their metabolic capacities.

\section{Conclusions}

In summary, we performed a systematic study of the local regulation of FOS metabolism in L. plantarum. The inactivation of sacR1 and sacR2 impaired the growth of $L$. plantarum and the metabolite formation in cultures containing FOS or glucose, respectively. The RT-qPCR data demonstrated SacR1 and SacR2 inhibited the expression of genes relevant to FOS metabolism in the absence of FOS, and these genes could be induced or derepressed by FOS. Furthermore, we predicted four potential TFBSs for SacR1 and SacR2 binding in multiple regions of the two FOS-related clusters in L. plantarum. We then verified the direct binding of SacR1 and SacR2 to these TFBSs in vitro and in vivo by EMSA and ChIP, respectively, which suggests that SacR1 and SacR2 act as local regulators through direct regulation of the transcription of FOS-related clusters. As local regulation is a component of FOS metabolism in L. plantarum, a further analysis of global and local regulation may give us a deeper understanding of the complex regulatory network underlying this metabolism. This information would serve as a theoretical basis upon which to construct an overall regulatory network of oligosaccharide metabolism by L. plantarum in vivo and could be also used as a model to study the utilization of oligosaccharides for other LAB.

\section{Methods}

\section{Bacterial strains, plasmids, oligonucleotides, and culture conditions}

The strains and plasmids used in the present study are summarized in Table 3. The oligonucleotide primers used are listed in Additional file 1: Table S1. Escherichia coli (E. coli) DH5 $\alpha$ and BL21, which were used for the cloning and/or expression of genes of interest, were propagated in Luria Bertani (LB) broth at $37{ }^{\circ} \mathrm{C}$ with aeration at $200 \mathrm{rpm} / \mathrm{min}$. L. plantarum ST-III and its mutant strains were cultivated anaerobically in deManRogosa-Sharpe (MRS) broth (Merck, Darmstadt, Germany) at $37^{\circ} \mathrm{C}$ without aeration. Where appropriate, the culture medium was supplemented with antibiotics at the following concentrations. To select antibiotic-resistant strains of E. coli, $100 \mu \mathrm{g} / \mathrm{mL}$ kanamycin, $50 \mu \mathrm{g} / \mathrm{mL}$ ampicillin, $30 \mu \mathrm{g} / \mathrm{mL}$ chloramphenicol, and $250 \mu \mathrm{g} / \mathrm{mL}$ erythromycin were added to LB. To select mutant strains of $L$. plantarum, $10 \mu \mathrm{g} / \mathrm{mL}$ chloramphenicol and 10 or $30 \mu \mathrm{g} /$ $\mathrm{mL}$ (for replica plating) erythromycin were added to MRS medium. 
Table 3 Strains and plasmids used in this study

\begin{tabular}{|c|c|c|}
\hline Strain and plasmid & Relevant feature & Source or reference \\
\hline \multicolumn{3}{|l|}{ Strains } \\
\hline L.plantarum & & CGMCC 0847 \\
\hline ST-III & Wild type & \\
\hline$\triangle \operatorname{sac} R 1:: \mathrm{cat}$ & Derivative of ST-III containing a lox66-P32-cat-lox71 replacement of sacR1 & This study \\
\hline$\triangle$ sacR2::cat & Derivative of ST-III containing a lox66-P32-cat-lox71 replacement of sacR2 & This study \\
\hline$\triangle \mathrm{sac} R 1$ & Derivative of ST-III containing a lox72 replacement of sacR1 & This study \\
\hline$\triangle s a c R 2$ & Derivative of ST-III containing a lox72 replacement of sacR2 & This study \\
\hline 409-Flag-sacR1 & Derivative of ST-III harboring pSIP409-Flag-sacR1 & This study \\
\hline 409-Flag-sacR2 & Derivative of ST-III harboring pSIP409-Flag-sacR2 & This study \\
\hline \multicolumn{3}{|l|}{ E. coli } \\
\hline $\mathrm{DH} 5 \mathrm{a}$ & For general gene cloning and plasmid construction & Promega \\
\hline BL21 & For protein expression & Novagen \\
\hline BL21-sacR1 & E. coli BL21 (DE3) harboring pTolo-EX5-sacR1 & This study \\
\hline BL21-sacR2 & E. coli BL21 (DE3) harboring Pet28a-sacR2 & This study \\
\hline \multicolumn{3}{|l|}{ Plasmid } \\
\hline pTolo-EX5 & $A p^{R}$, for cloning and protein expression, included His-tag & Tolobio \\
\hline pET-28a $(+)$ & $K_{a n a}{ }^{R}$, for cloning and protein expression, included His-tag & Novagen \\
\hline pTolo-EX5-sacR1 & $A p^{R}$, pTolo-EX5 with sacR1 gene cloned into Xhol sites & This study \\
\hline pET-28-sacR2 & Kana $^{R}$, pET-28a (+) with sacR2 gene cloned into Nhel/HindШ sites & This study \\
\hline pNZ5319 & $\mathrm{Cm}^{\mathrm{R}}, \mathrm{Em}^{\mathrm{R}}$; for multiple gene replacements in Gram-positive bacteria & [29] \\
\hline pNZ5319-up-down-1 & $\mathrm{Cm}^{\mathrm{R}}, \mathrm{Em}^{\mathrm{R}}$; pNZ5319 derivative containing homologous regions up and downstream of sacR1 & This study \\
\hline pNZ5319-up-down-2 & $\mathrm{Cm}^{\mathrm{R}}, \mathrm{Em}^{\mathrm{R}}$; pNZ5319 derivative containing homologous regions up and downstream of sacR2 & This study \\
\hline pNZ5348 & $\mathrm{Em}^{\mathrm{R}}$; contains cre under the control of the lp_1144 promoter & [29] \\
\hline pSIP409 & $\mathrm{Em}^{\mathrm{R}}$; for shuttle vector in E.coil, gusA controlled by $\mathrm{P}_{\text {sppQ }}$ & [53] \\
\hline pSIP409-Flag-sacR1 & $\mathrm{Em}^{\mathrm{R}} ; \mathrm{pSIP409}$ derivative; gusA replaced by Flag-tagged sacR1 & This study \\
\hline pSIP409-Flag-sacR2 & $\mathrm{Em}^{\mathrm{R}}$; pSIP409 derivative; gusA replaced by Flag-tagged sacR2 & This study \\
\hline
\end{tabular}

Kana ${ }^{R}$ kanamycin resistant; $A p^{R}$ ampicillin resistant, $\mathrm{Cm}^{R}$ chloramphenicol resistant, $E m^{R}$ erythromycin resistant

\section{Construction of sacR 1 and sacR2 mutants}

The L. plantarum ST-III deletion strain was generated using the Cre-lox-based mutagenesis system [31]. The upstream and downstream DNA regions of sacR1 and $s a c R 2$ were amplified using the respective primer pairs (Additional file 1: Table S1). The resultant DNA fragments were cloned into the suicide vector pNZ5319 to yield the pNZ5319-up-down-1 and pNZ5319-up-down-2 plasmid constructs. These deletion plasmids were transfected into L. plantarum ST-III cells via electroporation, and deletion mutants were screened as described previously [12, 34]. Candidate double-crossover clones were confirmed by PCR analysis.

\section{Growth analysis and detection of metabolites under different carbon sources}

For growth analysis, overnight cultures of L. plantarum ST-III and two deletion strains ( $\Delta s a c R 1$ and $\Delta s a c R 2)$ were transferred with $2 \%(\mathrm{v} / \mathrm{v})$ inoculum into $500 \mathrm{~mL}$ of CDM supplemented with filter-sterilized solutions of $1 \%$ (w/v) glucose, FOS (Meiji Seika Kaisha, Tokyo, Japan) or GOS (QuantumHi-Tech Biological Co., Ltd., Guangdong, China). The cultures were incubated for $16-18 \mathrm{~h}$ at $37{ }^{\circ} \mathrm{C}$ in a bioreactor (Bioflo model 115, New Brunswick Scientific Co., Edison, NJ, USA) and flushed with sterile air $(0.1 \mathrm{v} / \mathrm{v} \mathrm{min})$, without agitation and controlling the value of $\mathrm{pH}$. During the cells' growth up to the stationary phase, the samples were withdrawn every $2 \mathrm{~h}$ to measure the optical density at $600 \mathrm{~nm}\left(\mathrm{OD}_{600}\right)$ for growth analysis. The values of $\mu_{\max }$ were calculated through linear regressions of the plots of $\ln \left(\mathrm{OD}_{600}\right)$ versus time during the exponential growth phase [35]. When the $\mathrm{OD}_{600}$ reached 0.65 (early logarithmic phase), cultures were harvested by centrifugation $\left(8,000 \times g, 10 \mathrm{~min}, 4{ }^{\circ} \mathrm{C}\right)$. The supernatants were filtered through a $0.22 \mu \mathrm{m}$ nylon filter (Titan, China). The production of main metabolites was analyzed by high-performance liquid chromatography (HPLC) respectively, as previously reported [12]. 


\section{RNA extraction and RT-qPCR analysis}

FOS and GOS were selected as carbon sources for RNA extraction. The cultures were prepared as mentioned above and total RNA was extracted from exponentially growing wild-type and mutant cells $\left(\mathrm{OD}_{600}\right.$ of 0.65$)$ using TRIzol reagent (Invitrogen, Shanghai, China), as described previously [12]. Total RNA was then incubated with RNase-free DNase I and purified using a PrimeScript RT reagent kit (Takara Bio, Dalian, China). The quality and quantity of the RNA were evaluated using a Thermo Scientific Nanodrop 2000 device (Thermo, Waltham, MA, U.S.A.) and an Agilent 2100 Bioanalyzer (Agilent, Palo Alto, CA, U.S.A.), respectively.

For the RT-qPCR analysis, single-stranded cDNA was synthesized from total RNA using PrimeScript reverse transcriptase (Takara Bio, Dalian, China) according to the standard protocol. This synthesized cDNA was then used as a template for quantitative RT-PCR analysis, as described previously [10]. The primers used for the analysis are listed in Additional file 1: Table S1. All reactions were performed on the 7300 Fast Real-Time PCR System (Applied Biosystems, U.S.A.) using previously reported PCR cycling conditions [35]. To standardize the results, the relative abundance of $16 \mathrm{~S}$ rRNA [47] was used as the internal standard, and the relative gene expression data were calculated and analyzed using the $2^{-\Delta \mathrm{Ct}}$ method [48].

\section{Prediction of the binding sites of SacR1 and SacR2}

RSAT was used to analyze the consensus motif of the TFBSs for SacR1 and SacR2. The motifs were identified by scanning all upstream regions in the genome of L. plantarum ST-III based on the profiles of gene binding sites (Lp_0188 and Lp_3221) in L. plantarum WCSF1 via the RegPrecise database [2]. A PFM was constructed to collect TFBSs, and putative TFBSs in the upstream regions of sacPTS1 and sacPTS26 clusters were searched. The scores of candidate sites were calculated as the sums of the positional nucleotide weights, as previously described [49], and values $>5$ were considered indicative of potential TFBSs of SacR1 and SacR2.

\section{Purification of SacR1 and SacR2 proteins expressed in E. coli}

Expression of the sacR1 gene to produce recombinant protein was performed using the pTolo-EX5 vector (TOLO Biotech, Shanghai, China). Briefly, a $981 \mathrm{bp}$ sequence of the sacR1 gene was PCR amplified using the primer pair sacR1-F and sacR1-R, which includes the same XhoI site at the $5^{\prime}$ end of the primers (Additional file 1: Table S1). Subsequently, the amplified DNA was digested by XhoI and inserted into the corresponding site of the pToloEX5 vector. A 1,002 bp sequence of the $s a c R 2$ gene was
PCR amplified using the primer pair sacR2-F and sacR2$\mathrm{R}$, which include the NheI and HindIII sites at the $5^{\prime}$ end of the primers, respectively (Additional file 1: Table S1). Expression of the $s a c R 2$ gene was achieved by digesting amplified DNA using the two restriction endonucleases, followed by insertion into the corresponding sites of the pET-28a $(+)$ expression vector. The resulting plasmids, pTolo-EX5-sacR1 and pET-28a-sacR2, contained the target gene fused to an $\mathrm{N}$-terminal His-tag sequence. The recombinant plasmids were transformed as described previously [50], and the strain harboring these plasmids were named E. coli BL21- sacR1 and E. coli BL21- sacR2.

E. coli BL21(DE3) cells transformed with the two recombinant plasmids were grown at $37{ }^{\circ} \mathrm{C}$ in $100 \mathrm{~mL}$ of LB medium supplemented with kanamycin $(150 \mu \mathrm{g} /$ $\mathrm{mL}$ ). When the $\mathrm{OD}_{600}$ reached 0.4-0.6, expression of the recombinant gene was induced by the addition of $1 \mathrm{mM}$ isopropyl-b-D-thioisopropyl-b-D-thiogalactoside (IPTG). After an $8 \mathrm{~h}$ incubation at $25^{\circ} \mathrm{C}$, the cells were harvested by centrifugation. The $\mathrm{His}_{6}$-tagged proteins were extracted and purified by nickel ion affinity chromatography on a Chelating Sepharose Fast Flow column (GE Healthcare, Waukesha, WI, U.S.A.) according to the manufacturer's instructions. The purified proteins were desalted and concentrated using Amicon Ultra-0.5 centrifugal filter devices (Millipore, Billerica, MA, U.S.A.). The resultant proteins were used in EMSAs.

\section{Electrophoretic mobility-shift assay (EMSA)}

EMSAs were performed using $1 \mathrm{nM}$ double-stranded DNA fragments (Ppts1 - sacA, Pagl4, and PsacR2, 200 bp) that were generated by PCR using specific primer pairs (Additional file 1: Table S1). The DNA fragments were located in the four promoter regions of the sacPTS1 and sacPTS26 clusters. The DNA probes were incubated with increasing quantities of the selected proteins in binding buffer (50 mM Tris- $\mathrm{HCl}, \mathrm{pH} 8.0 ; 100 \mathrm{mM} \mathrm{KCl} ; 2.5 \mathrm{mM} \mathrm{MgCl}$, $0.2 \mathrm{mM}$ dithiothreitol [DTT]; $2 \mu \mathrm{g}$ polydIdC; $10 \%$ [v/v] glycerol) in a total reaction volume of $20 \mu \mathrm{L}$ for $30 \mathrm{~min}$ at $30{ }^{\circ} \mathrm{C}$. The samples were loaded onto $2 \%$ agarose gels containing $0.5 \times$ Tris-borate-EDTA buffer (TBE). To verify the specific binding of SacR1 and SacR2 to the TFBSs, each putative TFBS generated from the RSAT analysis according to the consensus motif was mutated and named TFBS-MUT (Additional file 1: Table S2). The mutations were introduced as previously reported [12].

\section{Chromatin immunoprecipitation assay (ChIP)}

The respective $s a c R 1$ and $s a c R 2$ overexpression plasmids pSIP409-Flag-sacR1 and pSIP409-Flag-sacR2 were constructed by inserting the purified sacR1 or sacR2 coding sequence into a restriction enzyme-digested pSIP409 vector as described previously (Additional file 1: Table S1) 
[50]. Next, the recombinant plasmids were electroporated into $L$. plantarum ST-III, which were used to produce 409-Flag-sacR1 and 409-Flag-sacR2 for ChIP.

The ChIP procedure was modified from existing protocols [12]. Briefly, for the strains 409-Flag-sacR1 and 409-Flag-sacR2, the cells were cultured at an $\mathrm{OD}_{600}$ of 0.3 and then induced with peptide pheromone IP-673 (synthesized by Invitrogen, Shanghai, China) in a final concentration of $50 \mathrm{ng} / \mathrm{mL}$ and allowed to grow for $2 \mathrm{~h}$ at $37^{\circ} \mathrm{C}$. Subsequently, in vivo cross-linking in the cultures was performed using $1 \%(\mathrm{v} / \mathrm{v})$ formaldehyde for $20 \mathrm{~min}$, and subsequently quenched by the addition of glycine to a final concentration of $0.125 \mathrm{M}$ at room temperature for $5 \mathrm{~min}$. The bacterial cells were collected by centrifugation at $5000 \times \mathrm{g}$ and $4{ }^{\circ} \mathrm{C}$ for $5 \mathrm{~min}$ and washed twice with ice-cold $5 \mathrm{mM}$ Tris- $\mathrm{HCl}(\mathrm{pH}$ 8.0). The pellet was resuspended in $5 \mathrm{mM}$ Tris- $\mathrm{HCl}(\mathrm{pH} 8.0)$ containing $5 \mu \mathrm{L}$ of protease inhibitors. Bacterial chromatin was sheared by ultrasonic disintegration (Bioraptor plus, Diagenode, Belgium) for $5 \mathrm{~min}$ at $4{ }^{\circ} \mathrm{C}$ with input setting 6 . After centrifugation, $5 \mathrm{~mL}$ of supernatant were transferred to a fresh tube as the input sample, and the remaining supernatant was added to the FLAG-binding beads overnight at $4{ }^{\circ} \mathrm{C}$ on a rotating wheel. On the next day, the beads were removed from the supernatant via magnetic separation (DynaMag ${ }^{\mathrm{TM}}-2$, Invitrogen, UK). The beads were washed four times in wash buffer (500 mM EDTA, $5 \mathrm{M}$ $\mathrm{NaCl}, 1 \mathrm{M}$ Tri-HCl, pH 8.0) and resuspended in $200 \mu \mathrm{L}$ of elution buffer. The resulting supernatant was collected after magnetic bead separation, mixed with $5 \mathrm{M}$ $\mathrm{NaCl}$, and heated to $65^{\circ} \mathrm{C}$ for $12 \mathrm{~h}$ to reverse cross-links. DNA was purified via phenol:chloroform extraction and ethanol precipitation [51]. The purified DNA samples were analyzed by qPCR using specific primers (Additional file 1: Table S1). Normal rabbit IgG was used as a negative control. All qPCRs were performed on the 7300 Fast Real-Time PCR System (Applied Biosystems) using a three-step PCR procedure (initial denaturation at $95{ }^{\circ} \mathrm{C}$ for $30 \mathrm{~s}$, followed by 40 cycles of denaturation at $95{ }^{\circ} \mathrm{C}$ for $5 \mathrm{~s}$, annealing at $54{ }^{\circ} \mathrm{C}$ for $25 \mathrm{~s}$, and synthesis at $60{ }^{\circ} \mathrm{C}$ for $25 \mathrm{~s}$ ). Product specificity was confirmed by a melting curve analysis. The qPCR results of each ChIP sample were normalized to a region of the 16S rRNA gene. Relative target levels were calculated using the fold enrichment method [52]. The results are reported as the average enrichment for three biological replicates.

\section{Statistical analysis}

The data shown herein are representative of at least three independent experiments. Student's t-test was used to determine statistical differences. Differences between values with $\mathrm{P}<0.05$ were considered statistically significant.

\section{Supplementary information}

Supplementary information accompanies this paper at https://doi. org/10.1186/s12934-020-01403-3.

Additional file 1: Table S1 Primers used in this study. Table S2 Nucleotide sequences of oligonucleotides harboring the putative transcription factor binding sites (TFBSs) and mutated sites used for electrophoretic mobility shift assays (EMSAs)

\section{Abbreviations \\ FOS: Fructooligosaccharides; TFBS: Transcription factor binding site; GIT: Gastrointestinal tract; LAB: Lactic acid bacteria; CCR: Carbon catabolite repres- sion; CcpA: Catabolite control protein A; cre: Catabolite responsive elements; GOS: Galactooligosaccharides; RT-qPCR: Reverse transcription-quantitative PCR; CDM: Chemically defined medium; EMSA: Electrophoretic mobility shift assay; ChIP: Chromatin immunoprecipitation; $\mu_{\max }$ : Maximal growth rate; PFM: Positional frequency matrix; RSAT: Regulatory Sequence Analysis Tools; AOS: Arabino-oligosaccharide; E. coli: Escherichia coli; LB: Luria Bertani; MRS: De Man-Rogosa-Sharpe; OD: Optical density; HPLC: High-performance liquid chromatography; IPTG: Isopropyl-b-D-thioisopropyl-b-D-thiogalactoside; TBE: Tris-borate-EDTA buffer.}

\section{Acknowledgements}

The authors are grateful to Dr Qiyao Wang and his students of East China University of Science and Technology for their help in ChIP-qPCR experiments.

\section{Authors' contributions}

CC wrote the manuscript and the statistical analysis. LW analyzed the growth and expression of related genes in wild-type and mutated strains. HY executed the target genes structure analysis and confirmed SacR1, SacR2 binding to the putative DNA sites. HT designed the research. All authors read and approved the final manuscript.

\section{Funding}

This work was supported by the National Natural Science Foundation of China (Award No. 31501451) and Shanghai Gaofeng \& Gaoyuan Project for University Academic Program Development.

\section{Availability of data and materials}

All data generated or analyzed during this study are included in this article and its additional file.

Ethics approval and consent to participate

Not applicable.

Consent for publication

Not applicable.

Competing interests

The authors declare that they have no competing interests.

Received: 14 February 2020 Accepted: 13 July 2020

Published online: 10 August 2020

\section{References}

1. Zhao H, Liu L, Peng S, Yuan L, Li H, Wang H. Heterologous expression of argininosuccinate synthase from Oenococcus oeni enhances the acid resistance of Lactobacillus plantarum. Front Microbiol. 2019;10:1393. https ://doi.org/10.3389/fmicb.2019.01393.

2. Martino ME, Bayjanov JR, Caffrey BE, Wels M, Joncour P, Hughes S, et al. Nomadic lifestyle of Lactobacillus plantarum revealed by comparative genomics of 54 strains isolated from different habitats. Environ Microbiol. 2016;18(12):4974-89. https://doi.org/10.1111/1462-2920.13455.

3. Giri SS, Sen SS, Saha S, Venkatachalam S, Park SC. Use of a potential probiotic, Lactobacillus plantarum L7, for the preparation of a 
rice-based fermented beverage. Front Microbiol. 2018;9:473. https://doi. org/10.3389/fmicb.2018.00473.

4. Goh YJ, Klaenhammer TR. Genetic mechanisms of prebiotic oligosaccharide metabolism in probiotic microbes. Annu Rev Food Sci Technol. 2015;6(6):137-56. https://doi.org/10.1146/annurev-food-022814-015706.

5. Capozzi V, Russo P, Ladero V, Fernández M, Fiocco D, Alvarez MA, et al. Biogenic amines degradation by Lactobacillus plantarum: toward a potential application in wine. Front Microbiol. 2012;3(122):122. https:// doi.org/10.3389/fmicb.2012.00122.

6. Gänzle MG, Rainer F. Metabolism of oligosaccharides and starch in Lactobacilli: a review. Front Microbiol. 2012;3:340. https://doi.org/10.3389/ fmicb.2012.00340.

7. Kant R, Blom J, Palva A, Siezen RJ, De Vos WM. Comparative genomics of Lactobacillus. Microb Biotechnol. 2011;4(3):323-32. https://doi.org/10.111 1/j.1751-7915.2010.00215.x.

8. Kaplan $\mathrm{H}$, Hutkins RW. Fermentation of fructooligosaccharides by lactic acid bacteria and bifidobacteria. Appl Environ Microbiol. 2000;66(6):2682. https://doi.org/10.1016/j.ijom.2009.11.012.

9. Ryan SM, Fitzgerald GF, Douwe VS. Transcriptional regulation and characterization of a novel beta-fructofuranosidase-encoding gene from Bifdobacterium breve UCC2003. Appl Environ Microbiol. 2005;71(7):3475-82. https://doi.org/10.1128/AEM.71.7.3475-3482.2005.

10. Lu Y, Song S, Tian H, Yu H, Zhao J, Chen C. Functional analysis of the role of CcpA in Lactobacillus plantarum grown on fructooligosaccharides or glucose: a transcriptomic perspective. Microb Cell Fact. 2018;17(1):201. https://doi.org/10.1186/s12934-018-1050-4.

11. Chen C, Guozhong Z, Wei C, Benheng G. Metabolism of fructooligosaccharides in Lactobacillus plantarum ST-III via differential gene transcription and alteration of cell membrane fluidity. Appl Environ Microbiol. 2015;81(22):7697-707. https://doi.org/10.1128/AEM.02426-15.

12. Chen C, Lu Y, Wang L, Yu H, Tian H. CcpA-dependent carbon catabolite repression regulates fructooligosaccharides metabolism in Lactobacillus plantarum. Front Microbiol. 2018;9:1114. https://doi.org/10.3389/fmicb 2018.01114.

13. Richard B, Stefanie M, Barbara S. Regulation of the $\beta$-hemolysin gene cluster of Streptococcus anginosus by CcpA. Sci Rep-UK. 2018;8(1):9028. https://doi.org/10.1038/s41598-018-27334-z.

14. Zhang C, Guo T, Xin Y, Gao X, Kong J. Catabolite responsive element deficiency of $x y /$ operon resulting in carbon catabolite derepression in Lactobacillus fermentum 1001. J Appl Microbiol. 2016;120(1):126-37. https ://doi.org/10.1111/jam.12990.

15. Akiyama T, Kimura $\mathrm{K}$, Hatano $\mathrm{H}$. Diverse galactooligosaccharides consumption by bifidobacteria: implications of $\beta$-galactosidase LacS operon. Biosci Biotech Bioch. 2015;79(4):664-72. https://doi.org/10.1080/09168 451.2014.987204.

16. Deutscher J. The mechanisms of carbon catabolite repression in bacteria. Curr Opin Microbiol. 2008;11(2):87. https://doi.org/10.1016/j. mib.2008.02.007.

17. Gorke B, Stulke J. Carbon catabolite repression in bacteria: many ways to make the most out of nutrients. Nat Rev Microbiol. 2008;6(8):613-24. https://doi.org/10.1038/nrmicro1932.

18. Panwar D, Kapoor M. Transcriptional analysis of galactomannooligosaccharides utilization by Lactobacillus plantarum WCFS1. Food Microbiol. 2020;86:103336. https://doi.org/10.1016/j.fm.2019.103336.

19. Muscariello L, Marasco R, De Felice M, Sacco M. The functional ccpA gene is required for carbon catabolite repression in Lactobacillus plantarum. Appl Environ Microbiol. 2001;67(7):2903-7. https://doi.org/10.1128/ AEM.67.7.2903-2907.2001.

20. Fujita Y. Carbon catabolite control of the metabolic network in Bacillus subtilis. J Agric Chemical Society of Japan. 2009;73(2):245-59. https://doi. org/10.1271/bbb.80479.

21. Tong H, Zeng L, Burne RA. The EllABMan phosphotransferase system permease regulates carbohydrate catabolite repression in Streptococcus gordonii. Appl Environ Microbiol. 2011;77(6):1957. https://doi.org/10.1128/ AEM.02385-10.

22. Lin Z, Burne RA. Seryl-phosphorylated HPr regulates CcpA-independent carbon catabolite repression in conjunction with PTS permeases in Streptococcus mutans. Mol Microbiol. 2010;75(5):1145-58. https://doi.org/ 10.1111/j.1365-2958.2009.07029.x.
23. Moye ZD, Lin Z, Burne RA. Fueling the caries process: carbohydrate metabolism and gene regulation by Streptococcus mutans. J Oral Microbiol. 2014;6(6):635-61. https://doi.org/10.3402/jom.v6.24878.

24. Zeng L, Chakraborty B, Farivar T, Burne RA. Coordinated regulation of the EllMan and fruRKI operons of Streptococcus mutans by global and fructose-specific pathways. Appl Environ Microbiol. 2017;83(21):e01403e1417. https://doi.org/10.1128/AEM.01403-17.

25. Monedero V, Mazé A, Boël G, Zúñiga M, Beaufils S, Hartke A, et al. The phosphotransferase system of lactobacillus casei: regulation of carbon metabolism and connection to cold shock response. J Mol Microb Biotech. 2007;12(1-2):20-322. https://doi.org/10.1159/000096456.

26. Francke C, Kerkhoven R, Wels M, Siezen RJ. A generic approach to identify transcription factor-specific operator motifs; inferences for Lacl-family mediated regulation in Lactobacillus plantarum WCFS1. BMC Genomics. 2008;9(1):145. https://doi.org/10.1186/1471-2164-9-145.

27. Goh YJ, Zhang C, Benson AK, Schlegel V, Lee J-H, Hutkins RW. Identification of a putative operon involved in fructooligosaccharide utilization by Lactobacillus paracasei. Appl Environ Microbiol. 2006;72(12):7518-30. https://doi.org/10.1128/aem.00877-06.

28. Andersen JM, Barrangou R, Hachem MA, Lahtinen S, Yong JG, Svensson $B$, et al. Transcriptional and functional analysis of galactooligosaccharide uptake by lacS in Lactobacillus acidophilus. Proc Natl Acad Sci USA. 2011;108(43):17785-90. https://doi.org/10.1073/pnas.1114152108.

29. Robert H, Le Marrec C, Blanco C, Jebbar M. Glycine betaine, carnitine, and choline enhance salinity tolerance and prevent the accumulation of sodium to a level inhibiting growth of Tetragenococcus halophila. Appl Environ Microbiol. 2000;66(2):509-17. https://doi.org/10.1128/ aem.66.2.509-517.2000.

30. Teusink B, Enckevort FHJV, Francke C, Wiersma A, Wegkamp A, Smid E, et al. In silico reconstruction of the metabolic pathways of Lactobacillus plantarum: comparing predictions of nutrient requirements with those from growth experiments. Appl Environ Microbiol. 2005;71(11):7253-62. https://doi.org/10.1128/AEM.71.11.7253-7262.2005.

31. Lambert JM, Bongers RS, Michiel K. Cre-lox-based system for multiple gene deletions and selectable-marker removal in Lactobacillus plantarum. Appl Environ Microbiol. 2007;73(4):1126. https://doi.org/10.1128/ AEM.01473-06.

32. Faustoferri RC, Hubbard CJ, Santiago B, Buckley AA, Quivey RG. Regulation of fatty acid biosynthesis by the global regulator CcpA and the local regulator FabT in Streptococcus mutans. Mol Oral Microbiol. 2014;30(2):128-46. https://doi.org/10.1111/omi.12076.

33. Wu Y, Yang Y, Ren C, Yang C, Yang S, Gu Y, et al. Molecular modulation of pleiotropic regulator CcpA for glucose and xylose coutilization by solvent-producing Clostridium acetobutylicum. Metab Eng. 2015;28:16979. https://doi.org/10.1016/j.ymben.2015.01.006.

34. Wang J, Guo H, Cao C, Wei Z, Kwok LY, Zhang H, et al. Characterization of the adaptive amoxicillin resistance of Lactobacillus casei Zhang by proteomic analysis. Front Microbiol. 2018;9:292. https://doi.org/10.3389/fmicb 2018.00292.

35. Chen C, Wang L, Lu Y, Yu H, Tian H. Comparative transcriptional analysis of Lactobacillus plantarum and its ccpA-knockout mutant under galactooligosaccharides and glucose conditions. Front Microbiol. 2019;10:1584. https://doi.org/10.3389/fmicb.2019.01584.

36. O"Connell KJ, O"Connell Motherway M, Liedtke A, Fitzgerald GF, Ross RP, Stanton C, et al. Transcription of two adjacent carbohydrate utilization gene clusters in Bifidobacterium breve UCC2003 is controlled by Lacl-and repressor open reading frame kinase (ROK)-type regulators. Appl Environ Microbiol. 2014;80(12):3604-14. https://doi.org/10.1128/AEM.00130-14.

37. Teixeira JS, Abdi R, Su MS-W, Schwab C, Gänzle MG. Functional characterization of sucrose phosphorylase and scrR a regulator of sucrose metabolism in Lactobacillus reuteri. Food Microbiol. 2013;36(2):432-9. https://doi. org/10.1016/j.fm.2013.07.011.

38. Alejandra MR, Cei AG, Morgane TC, Heladia S, Julio CV, Jacques VH. Theoretical and empirical quality assessment of transcription factor-binding motifs. Nucleic Acids Res. 2011;39(3):808-24. https://doi.org/10.1093/nar/ gkq710.

39. Almengor AC, Kinkel TL, Day SJ, Mciver KS. The catabolite control protein CcpA binds to Pmga and influences expression of the virulence regulator 
Mga in the Group A Streptococcus. J Bacteriol. 2007;189(23):8405-16. https://doi.org/10.1128/JB.01038-07.

40. Tiffert Y, Supra P, Wurm R, Wohlleben R, Wagner R, Reuther J. The Streptomyces coelicolor GInR regulon: identification of new GInR targets and evidence for a central role of $G \ln R$ in nitrogen metabolism in actinomycetes. Mol Microbiol. 2010;67(4):861-80. https://doi.org/10.111 1/j.1365-2958.2007.06092.x.

41. Jörg W, Astrid DG, Michael J, Peter VW, Ralph G. The CcpA regulon of Streptococcus suis reveals novel insights into the regulation of the streptococcal central carbon metabolism by binding of CcpA to two distinct binding motifs. Mol Microbiol. 2014;92(1):61-83. https://doi.org/10.1111/ mmi.12537.

42. Buntin N, Hongpattarakere T, Ritari J, Douillard FP, Paulin L, Boeren S, et al. An inducible operon is involved in inulin utilization in lactobacillus plantarum strains, as revealed by comparative proteogenomics and metabolic profiling. Appl Environ Microbiol. 2017;83(2):e02402-e2416. https://doi.org/10.1128/AEM.02402-16.

43. Xiong ZQ, Kong LH, Wang GQ, Xia YJ, Ai LZ. Functional analysis and heterologous expression of bifunctional glutathione synthetase from Lactobacillus. J Dairy Sci. 2018;101(8):6937-45. https://doi.org/10.3168/ jds.2017-14142.

44. Stevens MJ. Transcriptome response of Lactobacillus plantarum to global regulator deficiency, stress and other environmental conditions. Wageningen: Thesis Wageningen University; 2008.

45. Aleksandrzak-Piekarczyk T, Szatraj K, Kosiorek K. GlaR (YugA)-a novel RpiRfamily transcription activator of the Leloir pathway of galactose utilization in Lactococcus lactis IL1403. Microbiologyopen. 2019;8(5):e00714. https:// doi.org/10.1002/mbo3.714.

46. Arzamasov AA, van Douwe $\mathrm{S}$, Rodionov DA. Comparative genomics reveals the regulatory complexity of bifidobacterial arabinose and arabino-oligosaccharide utilization. Front Microbiol. 2018:9:776. https:// doi.org/10.3389/fmicb.2018.00776.
47. Zhao S, Zhang Q, Hao G, Liu X, Zhao J, Chen Y, et al. The protective role of glycine betaine in Lactobacillus plantarum ST-III against salt stress. Food Control. 2014;44:208-13. https://doi.org/10.1016/j.foodcont.2014.04.002.

48. Ren Q, Xu ZL, Wang XW, Zhao XF, Wang JX. Clip domain serine protease and its homolog respond to Vibrio challenge in Chinese white shrimp,Fenneropenaeus chinensis. Fish Shellfish Immun. 2009;26(5):78798. https://doi.org/10.1016/j.fsi.2009.03.004.

49. Sánchez B, Noriega L, Ruas-Madiedo P, de los Reyes-Gavilán CG, Margolles A. Acquired resistance to bile increases fructose-6-phosphate phosphoketolase activity in Bifidobacterium. FEMS Microbiol Lett. 2004;235:35-41. https://doi.org/10.1016/j.femsle.2004.04.009.

50. Chen C, Zhou F, Jing R, Ai L, Dong Y, Wu Z, et al. Cloning, expression and functional validation of a $\beta$-fructofuranosidase from Lactobacillus plantarum. Process Biochem. 2014;49(5):758-67. https://doi.org/10.1016/j. procbio.2014.02.013.

51. Russell DW, Sambrook J. Molecular cloning: a laboratory manual. Cold Spring Harbor: Cold Spring Harbor Laboratory Press; 2001 $10.2307 / 1309366$

52. Lacazette E. A laboratory practical illustrating the use of the ChIP-qPCR method in a robust model: Estrogen receptor alpha immunoprecipitation using Mcf-7 culture cells. Biochem Mol Biol Edu. 2017;45(2):152-60. https ://doi.org/10.1002/bmb.20999.

53. Sørvig E, Mathiesen G, Naterstad K, Eijsink VG, Axelsson L. High-level, inducible gene expression in Lactobacillus sakei and Lactobacillus plantarum using versatile expression vectors. Microbiol. 2005;151:2439-49. https://doi.org/10.1099/mic.0.28084-0.

\section{Publisher's Note}

Springer Nature remains neutral with regard to jurisdictional claims in published maps and institutional affiliations.
Ready to submit your research? Choose BMC and benefit from:

- fast, convenient online submission

- thorough peer review by experienced researchers in your field

- rapid publication on acceptance

- support for research data, including large and complex data types

- gold Open Access which fosters wider collaboration and increased citations

- maximum visibility for your research: over $100 \mathrm{M}$ website views per year

At BMC, research is always in progress.

Learn more biomedcentral.com/submissions 